\title{
Robust Fault Isolation With Statistical Uncertainty in Identified Parameters
}

\author{
Jianfei Dong, Michel Verhaegen and Fredrik Gustafsson
}

\section{Linköping University Post Print}

N.B.: When citing this work, cite the original article.

(C2012 IEEE. Personal use of this material is permitted. However, permission to reprint/republish this material for advertising or promotional purposes or for creating new collective works for resale or redistribution to servers or lists, or to reuse any copyrighted component of this work in other works must be obtained from the IEEE.

Jianfei Dong, Michel Verhaegen and Fredrik Gustafsson, Robust Fault Isolation With Statistical Uncertainty in Identified Parameters, 2012, IEEE Transactions on Signal Processing, (60), 10, 5556-5561. http://dx.doi.org/10.1109/TSP.2012.2208639

Postprint available at: Linköping University Electronic Press

http://urn.kb.se/resolve?urn=urn:nbn:se:liu:diva-84882 


\title{
Robust Fault Isolation with Statistical Uncertainty in Identified Parameters
}

\author{
Jianfei Dong, Michel Verhaegen, and Fredrik Gustafsson
}

\begin{abstract}
This paper is a companion paper to [1] and extends [1] to fault isolation. Also here use is made of a linear in the parameters model representation of the input-output behavior of the nominal system (i.e. fault-free). The projection of the residual onto directions only sensitive to individual faults is robustified against the stochastic errors of the estimated model parameters. The paper considers additive error sequences to the input and output quantities, that represent failures like drift, biased, stuck or saturated sensors/actuators.
\end{abstract}

\section{Index Terms}

Fault isolation; Parameter uncertainty; Statistical analysis; Additive faults; Closed-form solution.

\section{INTRODUCTION}

In classical FDI literature, fault isolation is usually enabled by projecting a residual vector onto the left null space of all but one fault input directions in the matrix that maps faults to outputs (referred to as fault transfer matrix in what follows), e.g. [2], [3]. But if these projection vectors are identified from data as in [3], it is difficult to quantify the statistical distribution of this solution against identification uncertainty. In this paper, we develop a new optimization-based solution, which searches for the projection directions in the subspace spanned by the non-principal components of the error covariance matrix of the identified fault transfer matrix. In other words, the residual vectors are projected onto the least variant subspace of the error covariance matrix, where the components of the identified parameters are most likely to be close to the their true values.

Corresponding author J. Dong, email: jfeidong@ hotmail.com. J. Dong was with Delft University of Technology when this work was carried out, and was with Philips Research, 5656 AE, Eindhoven, The Netherlands. M. Verhaegen is with Delft University of Technology, 2628CD, Delft, the Netherlands. F. Gustafsson is with Department of Electrical Engineering, Linköpings Universitet, SE-581 83 Linköping, Sweden. 
The rest of the paper is organized as follows. We start in Sec. II with the preliminaries and problem formulation. Sec. III goes further to derive a closed-from optimal isolation solution against the parameter identification errors. Sec. IV shows the improvements in fault isolation performance by our robustified method on aircraft dynamics. The notations in this paper are the same as those defined in [1, Sec. II.A].

\section{Preliminaries AND Problem Formulation}

\section{A. Fault isolation connected to the VARX description}

We consider the following discrete-time state-space model with additive faults:

$$
\begin{aligned}
x(k+1) & =A x(k)+B\left[u(k)+f_{a}(k)\right]+F w(k), \\
y(k) & =C x(k)+f_{s}(k)+v(k) .
\end{aligned}
$$

Here, $x(k) \in \mathbb{R}^{n}, y(k) \in \mathbb{R}^{\ell}, u(k) \in \mathbb{R}^{m}$, and $f_{a}(k) \in \mathbb{R}^{m}$ and $f_{s}(k) \in \mathbb{R}^{\ell}$ respectively stand for additive actuator and sensor faults. For brevity, we will collect all the faults into $f(k) \triangleq\left[f_{a}^{T}(k), f_{s}^{T}(k)\right]^{T} \in \mathbb{R}^{m+\ell}$, and denote $n_{f} \triangleq m+\ell$. Compared with the more general model in [1, Eqs. $\left.(1,2)\right]$, the fault model here correspond to the case where $E=[B, 0]$ and $G=[0, I]$; i.e. actuator faults share the same input channels with the control signals, and sensor faults directly add to the output measurements. This model can describe many commonly encountered additive faults, e.g. drifted, biased, stuck, or saturated actuators and sensors. The advantage of this model is that the Markov parameters from $f(k)$ to $y(k)$ are equal to those from $u(k)$ and $y(k)$ to $y(k)$, and can hence be estimated from I/O data.

Under the existence conditions of the stabilizing Kalman gain $K$, as specified in [1, Assumption 1], a closed-loop observer form of $(1,2)$ is (with $\Phi \triangleq A-K C$ )

$$
\begin{aligned}
\hat{x}(k+1) & =\Phi \hat{x}(k)+B u(k)+\left[\begin{array}{ll}
B & -K
\end{array}\right] f(k)+K y(k), \\
y(k) & =C \hat{x}(k)+\left[\begin{array}{ll}
0 & I
\end{array}\right] f(k)+e(k) .
\end{aligned}
$$

Here, $e(k)$ is the innovation signal defined in [1, Sec. II.D], and has a covariance matrix $\Sigma_{e}$. As detailed in [1], a residual generator for fault detection along the detection horizon $[k-L+1, k]$ takes the form:

$$
\begin{aligned}
\boldsymbol{r}_{k, L} & =\left(I-\boldsymbol{T}_{y}^{L}\right) \boldsymbol{y}_{k, L}-\boldsymbol{H}_{z}^{L, p} \boldsymbol{z}_{k-L, p}-\boldsymbol{T}_{u}^{L} \boldsymbol{u}_{k, L} \\
& =\boldsymbol{\varphi}_{f}+\boldsymbol{b}_{k, L}+\boldsymbol{e}_{k, L}
\end{aligned}
$$

To avoid repetition, we shall refer to [1] for the definition of the signal vectors $\boldsymbol{r}_{k, L}, \boldsymbol{u}_{k, L}, \boldsymbol{y}_{k, L}, \boldsymbol{b}_{k, L}, \boldsymbol{e}_{k, L}, \boldsymbol{\varphi}_{f}$ and the parametric matrices $\boldsymbol{H}_{z}^{L, p}, \boldsymbol{T}_{u}^{L}, \boldsymbol{T}_{y}^{L}$. We also denote these matrices with identified parameters by a bar on their top, e.g. $\overline{\boldsymbol{H}}_{z}^{L, p}$. It is useful to recall that $\boldsymbol{\varphi}_{f}=\left[\boldsymbol{H}_{f}^{L, p} \boldsymbol{T}_{f}^{L}\right] \cdot \boldsymbol{f}_{k, p+L}$, with $\boldsymbol{f}_{k, p+L}=$ 
$\left[f^{T}(k-L-p+1), \cdots, f^{T}(k)\right]^{T}$. Due to its role in mapping the fault signals to the outputs, we shall call $\left[\boldsymbol{H}_{f}^{L, p} \boldsymbol{T}_{f}^{L}\right]$ fault transfer matrix, which will be explicitly specified later.

The fault detection method in [1] aims at detecting the change in the mean of $\boldsymbol{r}_{k, L}$ due to a nonzero $\varphi_{f}$. But to tell what components of the various sensors and actuators in the system are faulty, one needs to separate the contributions of these components to $\boldsymbol{r}_{k, L}$. Let $\mathbb{F}$ denote the index set of the fault channels, i.e. $\mathbb{F}=\left\{1, \cdots, n_{f}\right\}$, and $\mathbb{F} \backslash i=\left\{1, \cdots, i-1, i+1, \cdots, n_{f}\right\}$. Denote $f_{k, p+L}^{i}=\left[f_{i}(k-L-p+1), \cdots, f_{i}(k)\right]$, where $f_{i}(k)$ denotes the $i$-th fault component at instant $k$. Let $f_{k, p+L}^{\mathbb{F} \backslash i}$ be the leftover of $\boldsymbol{f}_{k, p+L}$, after all its elements appearing in $\boldsymbol{f}_{k, p+L}^{i}$ are removed. Similarly, let $\boldsymbol{H}_{f, i}^{L, p}$ and $\boldsymbol{T}_{f, i}^{L}$ contain the columns of respectively $\boldsymbol{H}_{f}^{L, p}$ and $\boldsymbol{T}_{f}^{L}$ that only correspond to the $i$-th fault; and $\boldsymbol{H}_{f, \mathbb{F} \backslash i}^{L, p}$ and $\boldsymbol{T}_{f, \mathbb{F} \backslash i}^{L}$ contain all the other columns in $\boldsymbol{H}_{f}^{L, p}$ and $\boldsymbol{T}_{f}^{L}$. Then, $\boldsymbol{\varphi}_{f}$ can be written as

$$
\boldsymbol{\varphi}_{f}=\left[\begin{array}{cc}
\boldsymbol{H}_{f, i}^{L, p} & \boldsymbol{T}_{f, i}^{L}
\end{array}\right] \cdot \boldsymbol{f}_{k, p+L}^{i}+\left[\begin{array}{ll}
\boldsymbol{H}_{f, \mathbb{F} \backslash i}^{L, p} & \boldsymbol{T}_{f, \mathbb{F} \backslash i}^{L}
\end{array}\right] \cdot \boldsymbol{f}_{k, p+L}^{\mathbb{F} \backslash i}
$$

Similar to classical parity space methods [2], [3], isolating $f_{k, p+L}^{i}$ from $f_{k, p+L}^{\mathbb{F} \backslash i}$ can be achieved by designing a projection vector $\boldsymbol{p}_{i} \in \mathbb{R}^{1 \times L \ell}$ such that

$$
\boldsymbol{p}_{i} \cdot\left[\begin{array}{cc}
\boldsymbol{H}_{f, \mathbb{F} \backslash i}^{L, p} & \boldsymbol{T}_{f, \mathbb{F} \backslash i}^{L}
\end{array}\right]=0 \text {, and } \boldsymbol{p}_{i} \cdot\left[\begin{array}{cc}
\boldsymbol{H}_{f, i}^{L, p} & \boldsymbol{T}_{f, i}^{L}
\end{array}\right] \neq 0 .
$$

Projecting $\boldsymbol{r}_{k, L}$ onto such a $\boldsymbol{p}_{i}$ hence results in

$$
\boldsymbol{r}_{k, L}^{(i)} \triangleq \boldsymbol{p}_{i} \cdot\left[\left(\boldsymbol{I}-\boldsymbol{T}_{y}^{L}\right) \boldsymbol{y}_{k, L}-\boldsymbol{H}_{z}^{L, p} \boldsymbol{z}_{k-L, p}-\boldsymbol{T}_{u}^{L} \boldsymbol{u}_{k, L}\right]=\boldsymbol{p}_{i} \cdot\left(\left[\begin{array}{cc}
\boldsymbol{H}_{f, i}^{L, p} & \boldsymbol{T}_{f, i}^{L}
\end{array}\right] \cdot \boldsymbol{f}_{k, p+L}^{i}+\boldsymbol{b}_{k, L}+\boldsymbol{e}_{k, L}\right),
$$

which is only sensitive to the $i$-th fault component. The existence condition for $\boldsymbol{p}_{i}$ satisfying (5) is that

$$
\operatorname{rank}\left(\left[\boldsymbol{H}_{f}^{L, p} \boldsymbol{T}_{f}^{L}\right]\right)>\operatorname{rank}\left(\left[\boldsymbol{H}_{f, \mathbb{F} \backslash i}^{L, p} \boldsymbol{T}_{f, \mathbb{F} \backslash i}^{L}\right]\right) .
$$

\section{B. Fault isolation design using uncertain identified parameters}

The residual generator (3) is realized by identified parameters in the companion paper [1]. Projecting such a residual vector onto a direction $\boldsymbol{p}_{i}$ to isolate the $i$-th fault, $i=1, \cdots, n_{f}$, can then be computed by

$$
\boldsymbol{r}_{k, L}^{(i)}=\boldsymbol{p}_{i} \cdot\left[\left(\boldsymbol{I}-\overline{\boldsymbol{T}}_{y}^{L}\right) \boldsymbol{y}_{k, L}-\overline{\boldsymbol{H}}_{z}^{L, p} \boldsymbol{z}_{k-L, p}-\overline{\boldsymbol{T}}_{u}^{L} \boldsymbol{u}_{k, L}\right] .
$$

To design $\boldsymbol{p}_{i}$, we first need to build and analyze the fault transfer matrix, $\left[\boldsymbol{H}_{f}^{L, p} \boldsymbol{T}_{f}^{L}\right]$. However, the true parameters, $\boldsymbol{H}_{f}^{L, p}$ and $\boldsymbol{T}_{f}^{L}$, are unknown, but can be constructed from the identified Markov parameters of the nominal plant; i.e. $\hat{\Xi}$ in $[1$, Eq. (16)]. Based on the model $(1,2)$ and its closed-loop observer form, 
$\left[\boldsymbol{H}_{f}^{L, p} \mid \boldsymbol{T}_{f}^{L}\right]$ is derived and explicitly reads as follows,

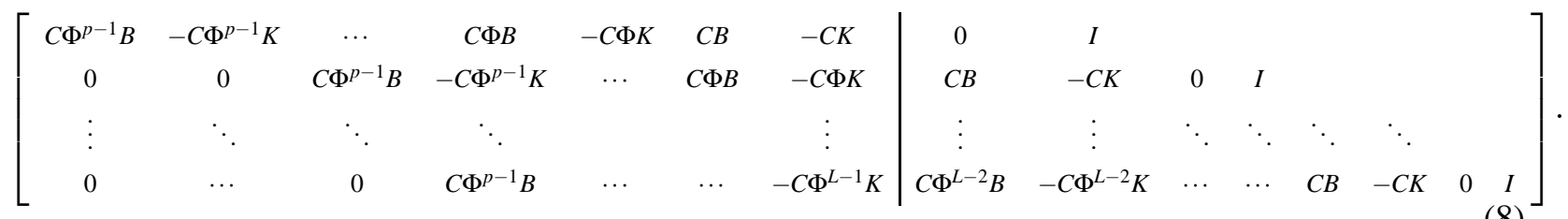

Then, based on the structure defined in (8), $\left[\boldsymbol{H}_{f}^{L, p} \boldsymbol{T}_{f}^{L}\right]$ can be constructed from the following sequence of parameters:

$$
\hat{\Xi}_{f}^{i d}=\left[\begin{array}{lllll}
\overline{C \Phi^{p-1} B} & -\overline{C \Phi^{p-1} K} & \ldots & \overline{C B} & -\overline{C K}
\end{array}\right]=\hat{\Xi} \cdot \operatorname{blockdiag}\left(I_{m},-I_{\ell}, \cdots, I_{m},-I_{\ell}\right) .
$$

Here, $\operatorname{blockdiag}\left(M_{1}, M_{2}\right)=\left[\begin{array}{ll}M_{1} & \\ & M_{2}\end{array}\right]$, for square matrices $M_{1}, M_{2}$. For brevity, denote

$$
\boldsymbol{I}_{\text {sgn }} \triangleq \operatorname{blockdiag}\left(I_{m},-I_{\ell}, \cdots, I_{m},-I_{\ell}\right) \in \mathbb{R}^{p(m+\ell) \times p(m+\ell)} .
$$

Note that due to the parameterization of $\left[\boldsymbol{H}_{f}^{L, p} \boldsymbol{T}_{f}^{L}\right]$ by the identified Markov parameters $\hat{\Xi}$, the fault transfer matrix $\left[\overline{\boldsymbol{H}}_{f}^{L, p} \overline{\boldsymbol{T}}_{f}^{L}\right]$ contains the errors $\Delta \hat{\Xi}$, as specified in $[1$, Eq. (17)]. We shall denote the following error matrix, $\left[\Delta \overline{\boldsymbol{H}}_{f}^{L, p} \Delta \overline{\boldsymbol{T}}_{f}^{L}\right]=\left[\boldsymbol{H}_{f}^{L, p} \boldsymbol{T}_{f}^{L}\right]-\left[\overline{\boldsymbol{H}}_{f}^{L, p} \overline{\boldsymbol{T}}_{f}^{L}\right]$, with a dimension of $\ell L \times(p+L)(m+$ $\ell)$. Due to the shifting structure expressed in (8), the errors in each block row of $\left[\Delta \overline{\boldsymbol{H}}_{f}^{L, p} \Delta \overline{\boldsymbol{T}}_{f}^{L}\right]$ is simply a repetition of the error source, $\Delta \hat{\Xi}$. We shall describe $\left[\Delta \overline{\boldsymbol{H}}_{f}^{L, p} \Delta \overline{\boldsymbol{T}}_{f}^{L}\right]$ in a compact form of $\Delta \hat{\Xi}$ with the shifting structure information. To this end, first note that due to the definition of $\hat{\Xi}_{f}^{i d}$ in (9), the errors in $\hat{\Xi}_{f}^{i d}$ can be written as, $\Delta \hat{\Xi}_{f}^{i d}=(\Xi-\hat{\Xi}) \cdot \boldsymbol{I}_{s g n}=\Delta \hat{\Xi} \cdot \boldsymbol{I}_{s g n}$.

On the other hand, $G=[0, I]$ is constant. $\left[\Delta \overline{\boldsymbol{H}}_{f}^{L, p} \Delta \overline{\boldsymbol{T}}_{f}^{L}\right]$ can hence be written as

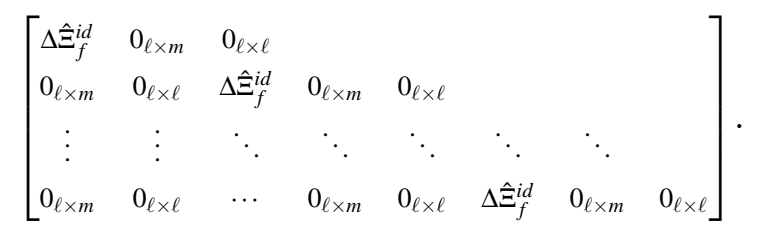

With some tedious but straightforward derivations, we can write this matrix in a compact form as

$$
\left(I_{L} \otimes \Delta \hat{\Xi}_{f}^{i d}\right) \cdot\left(S_{p, L} \otimes I_{m+\ell}\right)
$$

Here, $\boldsymbol{S}_{p, L}$ represents the following shifting matrix

$$
\boldsymbol{S}_{p, L}=\left[\begin{array}{ccc}
I_{p} & 0_{p \times 1} & 0_{p \times(L-1)} \\
0_{p \times 1} & I_{p} & 0_{p \times(L-1)} \\
& \vdots & \\
0_{p \times(L-1)} & I_{p} & 0_{p \times 1}
\end{array}\right] \in \mathbb{R}^{p L \times(p+L)},
$$

Now, the problem considered in this paper is as follows.

Problem 1: Determine a projection vector $\boldsymbol{p}_{i}\left(i=1, \cdots, n_{f}\right)$ that is only sensitive to the $i$-th fault component, and robust to the errors in the identified fault transfer matrix $\left[\overline{\boldsymbol{H}}_{f}^{L, p} \overline{\boldsymbol{T}}_{f}^{L}\right]$. 


\section{OPTIMAL ISOLATION ROBUST TO IDENTIFICATION UNCERTAINTY}

\section{A. Optimization-based solution to Problem 1}

A possible way to solve Problem 1 is via analyzing the statistical distribution of the residual in (7). But this is difficult. First, it is difficult to quantify in a closed-form the statistics of the left null space of $\left[\overline{\boldsymbol{H}}_{f, \mathbb{F} \backslash i}^{L, p} \overline{\boldsymbol{T}}_{f, \mathbb{F} \backslash i}^{L}\right]$ via SVD without any approximation. Second, even with an exact distribution function of this left null space, quantifying the distribution of $\boldsymbol{r}_{k, L}^{(i)}$ after the projection is still not easy, because the parameters in $\overline{\boldsymbol{T}}_{y}^{L}, \overline{\boldsymbol{H}}_{z}^{L, p}, \overline{\boldsymbol{T}}_{u}^{L}$ are also stochastic. We hence formulate a new optimization-based isolation problem against parameter errors.

Problem 2: Determine a projection vector $\boldsymbol{p}_{i}\left(i=1, \cdots, n_{f}\right)$ with $\left\|\boldsymbol{p}_{i}\right\|_{2}=1$ in the residual generator (7), which is optimal in the following sense:

O1. $\left\|\boldsymbol{p}_{i} \cdot\left[\begin{array}{cc}\overline{\boldsymbol{H}}_{f, i}^{L, p} & \overline{\boldsymbol{T}}_{f, i}^{L}\end{array}\right]\right\|_{2}^{2}$ is maximized;

O2. $\left\|\boldsymbol{p}_{i} \cdot\left[\begin{array}{cc}\overline{\boldsymbol{H}}_{f, \mathbb{F} \backslash i}^{L, p} & \overline{\boldsymbol{T}}_{f, \mathbb{F} \backslash i}^{L}\end{array}\right]\right\|_{2}^{2}$ is minimized;

O3. and the sensitivity of $\boldsymbol{p}_{i}$ to the following error covariance of the fault transfer matrix,

$$
\Sigma_{\Delta F T} \triangleq \mathbb{E}\left(\left[\Delta \overline{\boldsymbol{H}}_{f}^{L, p} \Delta \overline{\boldsymbol{T}}_{f}^{L}\right] \cdot\left[\Delta \overline{\boldsymbol{H}}_{f}^{L, p} \Delta \overline{\boldsymbol{T}}_{f}^{L}\right]^{T}\right),
$$

is also minimized; i.e. $\boldsymbol{p}_{i} \cdot \Sigma_{\Delta F T} \cdot \boldsymbol{p}_{i}^{T}$ is minimized.

A solution of $\boldsymbol{p}_{i}$ satisfying the first two objectives, i.e. O1 and O2, is actually a nominal design, which does not take into account the parameter errors. Similar nominal data-driven fault isolation design has been reported in [3]. The third objective, i.e. O3, aims at robustifying $\boldsymbol{p}_{i}$ against parameter errors.

Note that we choose to minimize $\boldsymbol{p}_{i} \cdot \Sigma_{\Delta F T} \cdot \boldsymbol{p}_{i}^{T}$, instead of directly minimizing $\boldsymbol{p}_{i} \cdot\left[\Delta \overline{\boldsymbol{H}}_{f}^{L, p} \Delta \overline{\boldsymbol{T}}_{f}^{L}\right]$, because the error matrix herein is unknown. But what we can indeed extract from data is its covariance information. On the other hand, $\boldsymbol{p}_{i} \cdot \Sigma_{\Delta F T} \cdot \boldsymbol{p}_{i}^{T}$ should ideally be zero. But this is unfortunately not possible, because by definition, the covariance matrix (12) is positive definite. Consequently, we can only find a $\boldsymbol{p}_{i}$, which is least sensitive to the covariance (12), instead of being strictly insensitive to it. This can be found by taking the subspace spanned by the vectors corresponding to the least $\operatorname{singular}$ values of $\Sigma_{\Delta F T}$; i.e. by the SVD

$$
\Sigma_{\Delta F T}=\left[\begin{array}{ll}
U_{p c}^{\Delta} & U_{n p c}^{\Delta}
\end{array}\right]\left[\begin{array}{ll}
S_{p c}^{\Delta} & \\
& S_{n p c}^{\Delta}
\end{array}\right]\left[\begin{array}{c}
\left(V_{p c}^{\Delta}\right)^{T} \\
\left(V_{n p c}^{\Delta}\right)^{T}
\end{array}\right] .
$$

Here, the subscripts "(n)pc" mean (non-)principal components. The diagonal matrix $S_{n p c}^{\Delta}$ contains the smallest $n_{\Delta}$ singular values of $\Sigma_{\Delta F T}$, with the integer $n_{\Delta}$ chosen by observing a gap among all the $\ell L$ singular values of $\Sigma_{\Delta F T}$. In subspace identification methods, e.g. [4], it is a standard practice to select model orders by observing singular values. 
Similarly, the projection direction satisfying $\mathrm{O} 2$ shall be in the subspace spanned by the vectors corresponding to the least singular values of

$$
Q_{i n s}^{f_{i}} \triangleq\left[\overline{\boldsymbol{H}}_{f, \mathbb{F} \backslash i}^{L, p} \overline{\boldsymbol{T}}_{f, \mathbb{F} \backslash i}^{L}\right] \cdot\left[\overline{\boldsymbol{H}}_{f, \mathbb{F} \backslash i}^{L, p} \overline{\boldsymbol{T}}_{f, \mathbb{F} \backslash i}^{L}\right]^{T} .
$$

Here, the subscripts "ins" are the abbreviation for insensitive.

The projection direction that satisfies both $\mathrm{O} 2$ and $\mathrm{O} 3$ is characterized in the following lemma.

Lemma 1: If range $\left(U_{n p c}^{\Delta}\right)$ is not orthogonal to $\operatorname{range}\left(Q_{i n s}^{f_{i}}\right)$, then the nonzero vector $\boldsymbol{p}_{i} \in \mathbb{R}^{1 \times \ell L}$ that simultaneously minimizes $\boldsymbol{p}_{i} \cdot Q_{i n s}^{f_{i}} \cdot \boldsymbol{p}_{i}^{T}$ and $\boldsymbol{p}_{i} \cdot \Sigma_{\Delta F T} \cdot \boldsymbol{p}_{i}^{T}$ is $\boldsymbol{p}_{i}^{T} \in \operatorname{range}\left(U_{n p c}^{\Delta} \cdot U_{n p c}^{\Delta, f_{i}}\right)$, where $U_{n p c}^{\Delta, f_{i}}$ is computed by the SVD

$$
\left(U_{n p c}^{\Delta}\right)^{T} \cdot Q_{i n s}^{f_{i}} \cdot U_{n p c}^{\Delta}=\left[\begin{array}{ll}
U_{p c}^{\Delta, f_{i}} & U_{n p c}^{\Delta, f_{i}}
\end{array}\right]\left[\begin{array}{ll}
S_{p c}^{\Delta, f_{i}} & \\
& S_{n p c}^{\Delta, f_{i}}
\end{array}\right]\left[\begin{array}{l}
\left(V_{p c}^{\Delta, f_{i}}\right)^{T} \\
\left(V_{n p c}^{\Delta, f_{i}}\right)^{T}
\end{array}\right],
$$

and has $n_{\Delta, f_{i}}$ columns, corresponding to the smallest $n_{\Delta, f_{i}}$ singular values of $\left(U_{n p c}^{\Delta}\right)^{T} \cdot Q_{i n s}^{f_{i}} \cdot U_{n p c}^{\Delta}$.

Proof: If $\boldsymbol{p}_{i}^{T} \in \operatorname{range}\left(U_{n p c}^{\Delta}\right)$, then $\exists \boldsymbol{\alpha} \in \mathbb{R}^{n_{\Delta}}$ such that $\boldsymbol{p}_{i}^{T}=U_{n p c}^{\Delta} \cdot \boldsymbol{\alpha}$. Now, $\boldsymbol{p}_{i} \cdot Q_{i n s}^{f_{i}} \cdot \boldsymbol{p}_{i}^{T}$ is reduced to

$$
\boldsymbol{\alpha}^{T} \cdot\left(U_{n p c}^{\Delta}\right)^{T} \cdot Q_{i n s}^{f_{i}} \cdot U_{n p c}^{\Delta} \cdot \boldsymbol{\alpha} .
$$

If range $\left(U_{n p c}^{\Delta}\right)$ is not orthogonal to range $\left(Q_{i n s}^{f_{i}}\right)$, then $\left(U_{n p c}^{\Delta}\right)^{T} \cdot Q_{i n s}^{f_{i}} \cdot U_{n p c}^{\Delta} \neq 0$, and (16) is minimized if $\boldsymbol{\alpha} \in \operatorname{range}\left(U_{n p c}^{\Delta, f_{i}}\right)$; i.e. $\exists \boldsymbol{\beta} \in \mathbb{R}^{n_{\Delta, f_{i}}}$ such that $\boldsymbol{\alpha}=U_{n p c}^{\Delta, f_{i}} \cdot \boldsymbol{\beta}$; and hence $\boldsymbol{p}_{i}^{T}=U_{n p c}^{\Delta} \cdot U_{n p c}^{\Delta, f_{i}} \cdot \boldsymbol{\beta}$ simultaneously minimizes $\boldsymbol{p}_{i} \cdot Q_{i n s}^{f_{i}} \cdot \boldsymbol{p}_{i}^{T}$ and $\boldsymbol{p}_{i} \cdot \Sigma_{\Delta F T} \cdot \boldsymbol{p}_{i}^{T}$. This is equivalent to $\boldsymbol{p}_{i}^{T} \in \operatorname{range}\left(U_{n p c}^{\Delta} \cdot U_{n p c}^{\Delta, f_{i}}\right)$.

For brevity, denote

$$
Q_{s e n}^{f_{i}} \triangleq\left[\begin{array}{cc}
\overline{\boldsymbol{H}}_{f, i}^{L, p} & \overline{\boldsymbol{T}}_{f, i}^{L}
\end{array}\right] \cdot\left[\begin{array}{cc}
\overline{\boldsymbol{H}}_{f, i}^{L, p} & \overline{\boldsymbol{T}}_{f, i}^{L}
\end{array}\right]^{T} .
$$

Here, the subscripts "sen" are the abbreviation for sensitive. Now, Problem 2 can be mathematically described by the following optimization problem:

$$
\begin{aligned}
\max _{\boldsymbol{p}_{i}} & \boldsymbol{p}_{i} \cdot Q_{s e n}^{f_{i}} \cdot \boldsymbol{p}_{i}^{T}, \\
\text { s.t. } & \boldsymbol{p}_{i}^{T} \in \operatorname{range}\left(U_{n p c}^{\Delta} \cdot U_{n p c}^{\Delta, f_{i}}\right), \text { and }\left\|\boldsymbol{p}_{i}\right\|_{2}=1 .
\end{aligned}
$$

The key challenge in solving the optimization problem (18) is to express the covariance matrix $\Sigma_{\Delta F T}$ in an explicit form of the covariance matrix of the identified $\hat{\Xi}$.

As analyzed in the companion paper [1], the bias effects of the initial states on both the parameter errors and the residual distribution can be neglected with a reasonably large past horizon $p$. Similarly, we assume that $p$ is large enough to ignore both $\boldsymbol{b}_{k, L}$ in (4), and the bias in the identified parameters. Then, following the discussions in [1, Sec. III.B], and as a standard practice in least squares, the covariance of 
the LS estimates of the Markov parameters in $\hat{\Xi}$ can be approximated by (where the strict equality holds with $N \rightarrow \infty$ ):

$$
\operatorname{Cov}(\operatorname{vec}(\Delta \hat{\Xi}))=\left(Z_{i d} Z_{i d}^{T}\right)^{-1} \otimes \Sigma_{e}
$$

which can be computed from the identification data matrix $Z_{i d}$ as defined in [1, Eq. (11)].

Theorem 1: Let the fault transfer matrix $\left[\begin{array}{cc}\overline{\boldsymbol{H}}_{f}^{L, p} & \overline{\boldsymbol{T}}_{f}^{L}\end{array}\right]$ be constructed from the identified Markov parameters $\hat{\Xi}$. Then,

$$
\begin{aligned}
& \mathbb{E}\left(\left[\begin{array}{ll}
\Delta \overline{\boldsymbol{H}}_{f}^{L, p} & \Delta \overline{\boldsymbol{T}}_{f}^{L}
\end{array}\right] \cdot\left[\begin{array}{cc}
\Delta \overline{\boldsymbol{H}}_{f}^{L, p} & \Delta \overline{\boldsymbol{T}}_{f}^{L}
\end{array}\right]^{T}\right)=\Pi_{1}-\Pi_{2}, \text { where } \\
\Pi_{1} & =\sum_{j=1}^{L}\left\{\operatorname{tr}\left[\left(P_{j} \otimes \boldsymbol{I}_{h}\right) \cdot\left(\boldsymbol{I}_{s g n}^{T}\left(\boldsymbol{Z}_{i d} \boldsymbol{Z}_{i d}^{T}\right)^{-1} \boldsymbol{I}_{s g n}\right)\right] \cdot\left[\left(W_{j}+W_{j}^{T}\right) \otimes \Sigma_{e}\right]\right\} \\
\Pi_{2} & =\operatorname{tr}\left(\boldsymbol{I}_{s g n}^{T}\left(\boldsymbol{Z}_{i d} \boldsymbol{Z}_{i d}^{T}\right)^{-1} \boldsymbol{I}_{s g n}\right) \cdot\left(\boldsymbol{I}_{L} \otimes \Sigma_{e}\right),
\end{aligned}
$$

with the structure matrices, $P_{j}$ and $W_{j}$, defined as

$$
P_{j}=\left[\begin{array}{ll}
0_{(j-1) \times(p-j+1)} & 0_{(j-1) \times(j-1)} \\
I_{p-j+1} & 0_{(p-j+1) \times(j-1)}
\end{array}\right] \in \mathbb{R}^{p \times p}, W_{j}=\left[\begin{array}{ll}
0_{(L-j+1) \times(j-1)} & I_{L-j+1} \\
0_{(j-1) \times(j-1)} & 0_{(j-1) \times(L-j+1)}
\end{array}\right] \in \mathbb{R}^{L \times L} .
$$

Proof: See Appendix A.

With Lemma 1 and Theorem 1, solving Problem 2, or equivalently (18), becomes straightforward, which is given in the following proposition.

Proposition 1: Let the fault transfer matrix $\left[\begin{array}{cc}\overline{\boldsymbol{H}}_{f}^{L, p} & \overline{\boldsymbol{T}}_{f}^{L}\end{array}\right]$ consist of the identified Markov parameters $\hat{\Xi}$. Then the optimal solution to (18) is $p_{i}=\omega_{i} \cdot\left(U_{n p c}^{\Delta} \cdot U_{n p c}^{\Delta, f_{i}}\right)^{T}$, where $\omega_{i}^{T}$ is the eigenvector that corresponds to the largest eigenvalue of the matrix, $\left(U_{n p c}^{\Delta} \cdot U_{n p c}^{\Delta, f_{i}}\right)^{T} \cdot Q_{s e n}^{f_{i}} \cdot\left(U_{n p c}^{\Delta} \cdot U_{n p c}^{\Delta, f_{i}}\right)$.

Remark 1: An alternative way of finding $\boldsymbol{p}_{i}$ that satisfies both $\mathrm{O} 2$ and $\mathrm{O} 3$ is seeking it in the subspace spanned by the non-principal components of $Q_{i n s}^{f_{i}}+\Sigma_{\Delta F T}$. This does not restrict the range of $Q_{i n s}^{f_{i}}$ from being orthogonal to that of $\Sigma_{\Delta F T}$, and is suitable for the case where the norms of these two matrices have comparable magnitudes, or the case of $\left\|\Sigma_{\Delta F T}\right\| \ll\left\|Q_{i n s}^{f_{i}}\right\|$, i.e. with negligible parameter errors.

As a summary, given $\hat{\Xi}$ and $\hat{\Sigma}_{e}$, five steps are needed to design the projection vectors, $\boldsymbol{p}_{i}, i=1, \cdots, n_{f}$.

1) Compute $\Sigma_{\Delta F T}$ by (20) and its SVD, and choose $U_{n p c}^{\Delta}$ by selecting its $n_{\Delta}$ smallest singular values.

2) Construct $\left[\overline{\boldsymbol{H}}_{f, i}^{L, p} \overline{\boldsymbol{T}}_{f, i}^{L}\right]$ and $\left[\overline{\boldsymbol{H}}_{f, \mathbb{F} \backslash i}^{L, p} \overline{\boldsymbol{T}}_{f, \mathbb{F} \backslash i}^{L}\right]$, and compute $Q_{i n s}^{f_{i}}, Q_{s e n}^{f_{i}}$, respectively by Eqs. $(14,17)$.

3) Compute the SVD of $\left(U_{n p c}^{\Delta}\right)^{T} \cdot Q_{i n s}^{f_{i}} \cdot U_{n p c}^{\Delta}$ and choose $U_{n p c}^{\Delta, f_{i}}$ by selecting its $n_{\Delta, f_{i}}$ smallest singular values.

4) Compute the eigenvector $\omega_{i}^{T}$ corresponding to the largest eigenvalue of $\left(U_{n p c}^{\Delta} U_{n p c}^{\Delta, f_{i}}\right)^{T} \cdot Q_{s e n}^{f_{i}} \cdot\left(U_{n p c}^{\Delta} U_{n p c}^{\Delta, f_{i}}\right)$.

5) Compute $\boldsymbol{p}_{i}=\boldsymbol{\omega}_{i} \cdot\left(U_{n p c}^{\Delta} \cdot U_{n p c}^{\Delta, f_{i}}\right)^{T}$. 


\section{B. Hypothesis-based fault isolation test}

With $\boldsymbol{p}_{i}$ solved by Proposition $1, \boldsymbol{r}_{k, L}^{(i)}$ has a maximum sensitivity to the $i$-th fault $f_{i}$, and minimum sensitivity to the other faults and to the parameter errors. Therefore, fault isolation can already be applied by choosing a threshold, which is large enough to upper bound the maximum amplitudes that the residual $\boldsymbol{r}_{k, L}^{(i)}$ can take in the fault-free case, see e.g. [5]. In this paper, we are interested in deriving a hypothesis test, where the threshold can be associated with a statistical confidence level.

The covariance of the residual vector $\boldsymbol{r}_{k, L}$ is derived and analyzed in details in the companion paper [1]. On the other hand, since $Q_{i n s}^{f_{i}}$ and $Q_{s e n}^{f_{i}}$ contain stochastic parameter errors, $\boldsymbol{p}_{i}$ as a solution of (18) is also stochastic. However, accounting the contribution of $\boldsymbol{p}_{i}$ to the variance of $\boldsymbol{r}_{k, L}^{(i)}$ requires deriving the probability distribution of the eigenvector of $\left(U_{n p c}^{\Delta} \cdot U_{n p c}^{\Delta, f_{i}}\right)^{T} \cdot Q_{s e n}^{f_{i}} \cdot\left(U_{n p c}^{\Delta} \cdot U_{n p c}^{\Delta, f_{i}}\right)$ that corresponds to its maximal eigenvalue, and then to consider the correlation between $\boldsymbol{p}_{i}$ and the parameter errors. The analysis therein may not even be tractable. But fortunately, by the formulation of Problem 2, the projection vector $\boldsymbol{p}_{i}$ is in the least variant subspace of the parameter errors. A reasonable approximation is therefore to treat $\boldsymbol{p}_{i}, i=1, \cdots, n_{f}$ as deterministic vectors, in determining the variance of $\boldsymbol{r}_{k, L}^{(i)}$.

As analyzed in the companion paper [1], with the past horizon $p$ chosen sufficiently large, $\operatorname{Cov}\left(\boldsymbol{r}_{k, L}\right)$ can be approximated by [1, Eq. (38)]; and in the fault-free case, the statistical distribution of $\boldsymbol{r}_{k, L}^{T} \mathrm{Cov}^{-1}\left(\boldsymbol{r}_{k, L}\right) \boldsymbol{r}_{k, L}$ can be described as a central $\chi^{2}$ distribution. Now, since $\boldsymbol{p}_{i}, i=1, \cdots, n_{f}$ are optimized such that they are least sensitive to the stochastic parameter errors, the variance of $\boldsymbol{r}_{k, L}^{(i)}$ can be approximated by $\boldsymbol{p}_{i} \cdot \operatorname{Cov}\left(\boldsymbol{r}_{k, L}\right) \cdot \boldsymbol{p}_{i}^{T}$; and fault isolation can be tested by a central $\chi^{2}$ test with 1 DoF, i.e.

$$
\tau_{i}(k)=\frac{\left(\boldsymbol{r}_{k, L}^{(i)}\right)^{2}}{\boldsymbol{p}_{i} \cdot \operatorname{Cov}\left(\boldsymbol{r}_{k, L}\right) \cdot \boldsymbol{p}_{i}^{T}} \underset{\substack{\text { faulty fault } \\ \gtrless}}{\gtrless},
$$

where $\gamma_{\alpha}$ is the threshold, with a false alarm rate (FAR) of $\alpha$.

\section{Simulation STUdies}

Consider the same VTOL model as studied in the companion paper [1]. The parameters in the simulation are all the same as those given in [1, Sec. IV.B.1], and are not repeated.

Since there are two actuator channels and four sensor channels, $n_{f}=6$. We hence designed six projection vectors for isolating each of these faults, using the data from one single identification experiment, as described in [1, Sec. IV.B.1]. These filters are denoted as F1, F2, F3, F4, F5, and F6, and respectively designed for isolating a fault signal that can affect one out of the six I/O channels, i.e. $u_{1}, u_{2}, y_{1}, y_{2}, y_{3}, y_{4}$. 
We considered similar additive actuator faults, as defined in [1, Sec. IV.B.2], but with an overlap in their effective intervals to test the fault isolation performance. The first actuator was stuck at -3 in the interval of $301 \leq k \leq 600$; and the second actuator had a bias of -5 in the interval of $501 \leq k \leq 800$.

We compared the results achieved by the robust solution in Proposition 1 to the following nominal design without considering the parameter errors, as similar to the isolation solution in [3]:

$$
\begin{aligned}
\max _{\boldsymbol{p}_{i}} & \left\|\boldsymbol{p}_{i} \cdot\left[\begin{array}{cc}
\overline{\boldsymbol{H}}_{f, i}^{L, p} & \overline{\boldsymbol{T}}_{f, i}^{L}
\end{array}\right]\right\|_{2}^{2}, i=1, \cdots, n_{f} \\
\text { s.t. } & \left\|\boldsymbol{p}_{i} \cdot\left[\begin{array}{cc}
\overline{\boldsymbol{H}}_{f, \mathbb{F} \backslash i}^{L, p} & \overline{\boldsymbol{T}}_{f, \mathbb{F} \backslash i}^{L}
\end{array}\right]\right\|_{2}^{2}=0, \text { and }\left\|\boldsymbol{p}_{i}\right\|_{2}=1 .
\end{aligned}
$$

Here, the fault transfer matrices were the same as used in the robust design. But in this nominal solution, only the innovation signals were considered in the residual variance. In the nominal filters, the orders of the non-principal subspace of $\left[\overline{\boldsymbol{H}}_{f, \mathbb{F} \backslash i}^{L, p} \overline{\boldsymbol{T}}_{f, \mathbb{F} \backslash i}^{L}\right], i=1, \cdots, 6$ were respectively chosen according to their singular values (40 in total) as 30 for F1 and F2, and 20 for F3 F6.

In the robust filters, the orders were chosen as $n_{\Delta}=30$ according to the singular values (40 in total) of $\Sigma_{\Delta F T}$, and as $n_{\Delta, f_{1}}, n_{\Delta, f_{2}}=20$ for F1 and F2 and $n_{\Delta, f_{i}}=10, i=3, \cdots, 6$ for F3 F6 according to the singular values (30 in total) of respectively $\left(U_{n p c}^{\Delta}\right)^{T} \cdot Q_{i n s}^{f_{i}} \cdot U_{n p c}^{\Delta}, i=1, \cdots, 6$. The results are illustrated in Fig. 1. Clearly, when no fault occurred in I/O channels, the robust isolation test reacted "almost" correctly, since the stochastic parameter errors were accounted in the residual variance. The slightly bigger false alarm rate of the robust filters compared to $0.5 \%$ can be attributed to the fact that the hypothesis test (21) is an approximation by treating $\boldsymbol{p}_{i}, i=1, \cdots, 6$ as deterministic. But since $\boldsymbol{p}_{i}$ is in the least variant subspace of the parameter errors, the approximation error is reasonably small. Moreover, the two faults were correctly isolated respectively by the robust filters F1 and F2, which were designed to be sensitive respectively only to the first and the second actuator.

In comparison, these results clearly outperformed those achieved by the nominal design, especially when the second actuator failed. The nominal filter F2 did not react at all to this fault. The nominal filters F3, F4, F5 all gave alarms in the interval $501 \leq k \leq 600$. Besides, F1 still gave alarms in the interval $601 \leq k \leq 800$, when the first actuator recovered from the stuck failure. This then denied the correct isolation of the two actuator faults.

\section{CONCLusions}

In this paper, we have developed a new data-driven fault isolation method, which is robust to parameter identification errors. The main contributions are the closed-form error covariance matrix of the identified fault transfer matrix, and the robustified fault isolation vectors that belong to the subspace spanned by the 

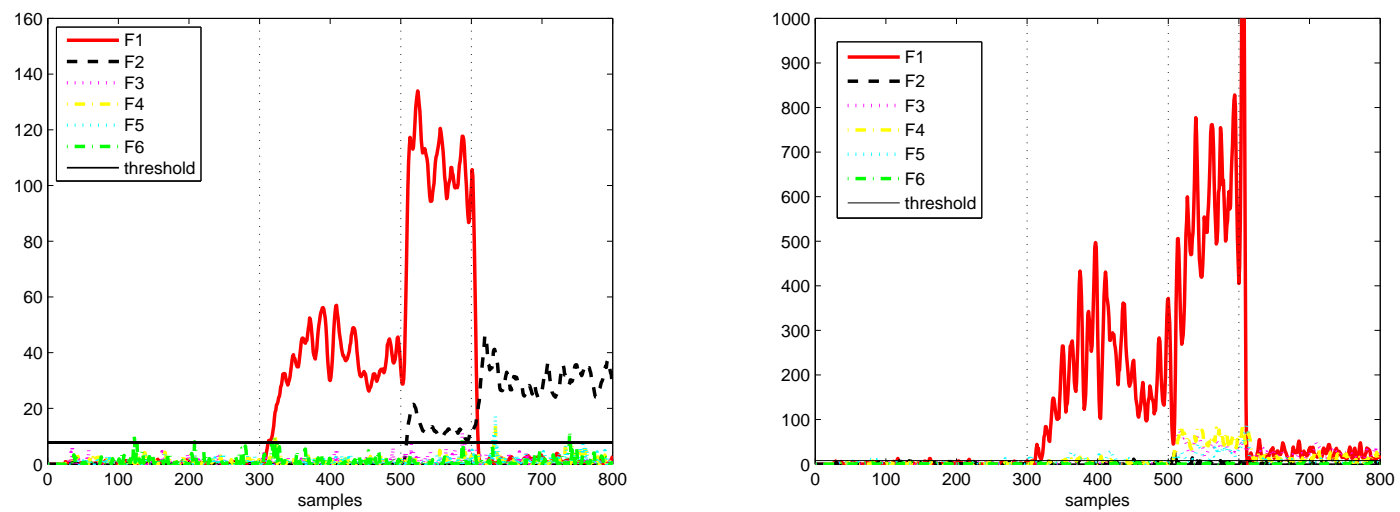

Fig. 1. Robust (left) v.s. nominal (right) fault isolation.

non-principal components of this covariance matrix. Our analytical results are tested in the simulation studies, which have validated that the data-driven fault isolation method developed in this paper has clearly improved performance compared to the nominal data-driven solution without taking into account the identification uncertainty. Possible future directions are to extend the robust isolation method to deal with multiplicative faults and to linear parameter varying systems.

\section{REFERENCES}

[1] J. Dong, M. Verhaegen, and F. Gustafsson, "Robust fault detection with statistical uncertainty in identified parameters," IEEE Transactions on Signal Processing, vol. 60, pp. 5064-5076, 2012.

[2] J. Gertler and D. Singer, "A new structural framework for parity equation based failure detection and isolation," Automatica, vol. 26, pp. 381-388, 1990.

[3] S. Qin and W. Li, "Detection and identification of faulty sensors in dynamic processes," AIChE Journal, vol. 47, pp. 1581-1593, 2001.

[4] A. Chiuso, "On the relation between CCA and predictor-based subspace identification," IEEE Transactions on Automatic Control, vol. 52, pp. 1795-1812, 2007.

[5] Y. Wang, S. Ding, H. Ye, and G. Wang, "A new fault detection scheme for networked control systems subject to uncertain time-varying delay," IEEE Transactions on Signal Processing, vol. 56, pp. 5258-5268, 2008.

\section{APPENDIX A}

\section{PROOF OF THEOREM 1}

Lemma 2: Let $\boldsymbol{S}_{p, L}$ be defined as in (11). Then,

$$
\boldsymbol{S}_{p, L} \cdot \boldsymbol{S}_{p, L}^{T}=\sum_{j=1}^{L}\left(W_{j} \otimes P_{j}+W_{j}^{T} \otimes P_{j}^{T}\right)-I_{p L}
$$


where the matrices, $P_{j}$ and $W_{j}$ for $j=1, \cdots, L$, are defined in Theorem 1 .

The derivation of this expression is lengthy and purely algebraic, and shall be omitted for brevity. It can be easily verified by a numerical simulation.

Lemma 3: Let $M \in \mathbb{R}^{n \times n}$. Then, $\operatorname{vec}^{T}\left(I_{n}\right) \cdot \operatorname{vec}(M)=\operatorname{tr}(M)$.

Proof: Let $\eta_{i}$ be the $i$-th column of $I_{n}$. Let $M_{i}$ denote the $i$-th column of $M$, and $M_{i i}$ be its $i$-th diagonal element. Then, $\operatorname{vec}^{T}\left(I_{n}\right) \cdot \operatorname{vec}(M)=\sum_{i=1}^{n} \eta_{i}^{T} \cdot M_{i}=\sum_{i=1}^{n} M_{i i}$, which equals $\operatorname{tr}(M)$, by definition.

Lemma 4: Let $M, \tilde{M} \in \mathbb{R}^{n \times n}$. Then, $\operatorname{vec}^{T}\left(I_{n}\right) \cdot(M \otimes \tilde{M}) \cdot \operatorname{vec}\left(I_{n}\right)=\operatorname{tr}\left(\tilde{M} \cdot M^{T}\right)$.

Proof: First, by [1, Property (43)], $(M \otimes \tilde{M}) \cdot \operatorname{vec}\left(I_{n}\right)=\operatorname{vec}\left(\tilde{M} \cdot I_{n} \cdot M^{T}\right)=\operatorname{vec}\left(\tilde{M} \cdot M^{T}\right)$. Now, by Lemma 3, $\operatorname{vec}^{T}\left(I_{n}\right) \cdot \operatorname{vec}\left(\tilde{M} \cdot M^{T}\right)=\operatorname{tr}\left(\tilde{M} \cdot M^{T}\right)$.

First, recall that $\left[\Delta \overline{\boldsymbol{H}}_{f}^{L, p} \Delta \overline{\boldsymbol{T}}_{f}^{L}\right]$ can be written in terms of the error sources $\Delta \hat{\vec{\Xi}}_{f}^{i d}$ and its shifting structure as $\left(I_{L} \otimes \Delta \hat{\Xi}_{f}^{i d}\right) \cdot\left(\boldsymbol{S}_{p, L} \otimes I_{m+\ell}\right)$, where $\Delta \hat{\Xi}_{f}^{i d}=\Delta \hat{\vec{\Xi}} \cdot \boldsymbol{I}_{s g n}$. Since we consider the general case of multiple outputs, i.e. $\ell>1, \Delta \hat{\Xi}$ is an $\ell \times p(m+\ell)$ matrix, instead of a vector. The covariance of this matrix-value random variable denoted as $\operatorname{Cov}(\operatorname{vec}(\Delta \hat{\bar{\Xi}}))$ is given in (19). It is straightforward to show

$$
\operatorname{Cov}\left(\operatorname{vec}\left(\Delta \hat{\Xi}_{f}^{i d}\right)\right)=\left[\boldsymbol{I}_{s g n}^{T} \cdot\left(\boldsymbol{Z}_{i d} \boldsymbol{Z}_{i d}^{T}\right)^{-1} \cdot \boldsymbol{I}_{s g n}\right] \otimes \Sigma_{e}
$$

To simplify notations in this proof, denote $\boldsymbol{\Theta}_{f} \triangleq \operatorname{vec}\left(\Delta \hat{\Xi}_{f}^{i d}\right) \in \mathbb{R}^{\ell p n_{f}}, \Omega \triangleq \boldsymbol{I}_{s g n}^{T} \cdot\left(\boldsymbol{Z}_{i d} \boldsymbol{Z}_{i d}^{T}\right)^{-1} \cdot \boldsymbol{I}_{\text {sgn }} \in$ $\mathbb{R}^{p n_{f} \times p n_{f}}$; i.e. (22) can be rewritten as $\operatorname{Cov}\left(\Theta_{f}\right)=\Omega \otimes \Sigma_{e}$.

Since $\boldsymbol{\Theta}_{f}=\operatorname{vec}\left(\Delta \hat{\Xi}_{f}^{i d}\right), \Delta \hat{\Xi}_{f}^{i d}$ can be retrieved from $\boldsymbol{\Theta}_{f}$ :

$$
\Delta \hat{\boldsymbol{\Xi}}_{f}^{i d}=\left[\begin{array}{llll}
\left(\eta_{1}^{T} \otimes \boldsymbol{I}_{\ell}\right) \cdot \boldsymbol{\Theta}_{f} & \left(\eta_{2}^{T} \otimes \boldsymbol{I}_{\ell}\right) \cdot \boldsymbol{\Theta}_{f} & \cdots & \left(\eta_{p n_{f}}^{T} \otimes \boldsymbol{I}_{\ell}\right) \cdot \boldsymbol{\Theta}_{f}
\end{array}\right]=\left[\begin{array}{l}
\overrightarrow{\boldsymbol{I}}_{p n_{f}}^{T} \otimes \boldsymbol{I}_{\ell}
\end{array}\right] \cdot\left(I_{p n_{f}} \otimes \boldsymbol{\Theta}_{f}\right),
$$

where $\eta_{i} \in \mathbb{R}^{p n_{f} \times 1}$ denotes the $i$-th column of $I_{p n_{f}}$. Thus, $\left[\Delta \overline{\boldsymbol{H}}_{f}^{L, p} \Delta \overline{\boldsymbol{T}}_{f}^{L}\right]$ can be explicitly written as

$$
\left\{I_{L} \otimes\left[\left(\overrightarrow{\boldsymbol{I}}_{p n_{f}}^{T} \otimes I_{\ell}\right) \cdot\left(I_{p n_{f}} \otimes \boldsymbol{\Theta}_{f}\right)\right]\right\} \cdot\left(\boldsymbol{S}_{p, L} \otimes I_{m+\ell}\right) .
$$

For simplicity, denote $\overrightarrow{\boldsymbol{I}}_{p n_{f}} \triangleq \operatorname{vec}\left(I_{p n_{f}}\right)$. By [1, Properties $\left.(45,46,47)\right]$, the error covariance of the fault transfer matrix can be written as

$$
\begin{aligned}
& \mathbb{E}\left(\left[\begin{array}{cc}
\Delta \overline{\boldsymbol{H}}_{f}^{L, p} & \Delta \overline{\boldsymbol{T}}_{f}^{L}
\end{array}\right] \cdot\left[\begin{array}{cc}
\Delta \overline{\boldsymbol{H}}_{f}^{L, p} & \Delta \overline{\boldsymbol{T}}_{f}^{L}
\end{array}\right]^{T}\right) \\
= & \mathbb{E}\left\{\left\{I_{L} \otimes\left[\left(\overrightarrow{\boldsymbol{I}}_{p n_{f}}^{T} \otimes I_{\ell}\right) \cdot\left(I_{p n_{f}} \otimes \boldsymbol{\Theta}_{f}\right)\right]\right\} \cdot\left(\boldsymbol{S}_{p, L} \otimes I_{n_{f}}\right) \cdot\left(\boldsymbol{S}_{p, L}^{T} \otimes I_{n_{f}}\right) \cdot\left\{I_{L} \otimes\left[\left(I_{p n_{f}} \otimes \boldsymbol{\Theta}_{f}^{T}\right) \cdot\left(\overrightarrow{\boldsymbol{I}}_{p n_{f}} \otimes I_{\ell}\right)\right]\right\}\right\} \\
= & \mathbb{E}\left\{\left\{\left\{I_{L} \otimes\left[\left(\overrightarrow{\boldsymbol{I}}_{p n_{f}}^{T} \otimes I_{\ell}\right) \cdot\left(I_{p n_{f}} \otimes \boldsymbol{\Theta}_{f}\right)\right]\right\} \cdot\left[\left(\boldsymbol{S}_{p, L} \boldsymbol{S}_{p, L}^{T}\right) \otimes I_{n_{f}}\right] \cdot\left\{I_{L} \otimes\left[\left(I_{p n_{f}} \otimes \boldsymbol{\Theta}_{f}^{T}\right) \cdot\left(\overrightarrow{\boldsymbol{I}}_{p n_{f}} \otimes I_{\ell}\right)\right]\right\}\right\} .\right.
\end{aligned}
$$

Now, the key idea is to explicitly obtain $\mathbb{E}\left(\Theta_{f} \cdot \Theta_{f}^{T}\right)$ in this equation and use (22). First note that $\boldsymbol{S}_{p, L} \boldsymbol{S}_{p, L}^{T} \in \mathbb{R}^{p L \times p L}$; but $\left(\overrightarrow{\boldsymbol{I}}_{p n_{f}}^{T} \otimes \boldsymbol{I}_{\ell}\right) \cdot\left(\boldsymbol{I}_{p n_{f}} \otimes \boldsymbol{\Theta}_{f}\right) \in \mathbb{R}^{\ell \times p n_{f}}$. We hence need Lemma 2. The equation 
above then boils down to

$\mathbb{E}\left\{\left\{I_{L} \otimes\left[\left(\overrightarrow{\boldsymbol{I}}_{p n_{f}}^{T} \otimes I_{\ell}\right) \cdot\left(I_{p n_{f}} \otimes \boldsymbol{\Theta}_{f}\right)\right]\right\} \cdot\left[\left(\sum_{j=1}^{L}\left(W_{j} \otimes P_{j}+W_{j}^{T} \otimes P_{j}^{T}\right)-I_{p L}\right) \otimes I_{n_{f}}\right] \cdot\left\{I_{L} \otimes\left[\left(I_{p n_{f}} \otimes \boldsymbol{\Theta}_{f}^{T}\right) \cdot\left(\overrightarrow{\boldsymbol{I}}_{p n_{f}} \otimes I_{\ell}\right)\right]\right\}\right\}$.

Let us first derive

$$
\mathbb{E}\left\{\left\{I_{L} \otimes\left[\left(\overrightarrow{\boldsymbol{I}}_{p n_{f}}^{T} \otimes I_{\ell}\right) \cdot\left(I_{p n_{f}} \otimes \boldsymbol{\Theta}_{f}\right)\right]\right\} \cdot\left[\left(\sum_{j=1}^{L} W_{j} \otimes P_{j}\right) \otimes I_{n_{f}}\right] \cdot\left\{I_{L} \otimes\left[\left(I_{p n_{f}} \otimes \boldsymbol{\Theta}_{f}^{T}\right) \cdot\left(\overrightarrow{\boldsymbol{I}}_{p n_{f}} \otimes I_{\ell}\right)\right]\right\}\right\} .
$$

Recall that $P_{j} \in \mathbb{R}^{p \times p}$ and $W_{j} \in \mathbb{R}^{L \times L}$ for $j=1, \cdots, L$. By [1, Properties $\left.(44,45)\right]$, Eq. (25) equals to

$$
\begin{aligned}
& \sum_{j=1}^{L} \mathbb{E}\left\{\left\{I_{L} \otimes\left[\left(\overrightarrow{\boldsymbol{I}}_{p n_{f}}^{T} \otimes I_{\ell}\right) \cdot\left(I_{p n_{f}} \otimes \boldsymbol{\Theta}_{f}\right)\right]\right\} \cdot\left(W_{j} \otimes P_{j} \otimes I_{n_{f}}\right) \cdot\left\{I_{L} \otimes\left[\left(I_{p n_{f}} \otimes \boldsymbol{\Theta}_{f}^{T}\right) \cdot\left(\overrightarrow{\boldsymbol{I}}_{p n_{f}} \otimes I_{\ell}\right)\right]\right\}\right\} \\
= & \sum_{j=1}^{L} \mathbb{E}\left\{\left\{W_{j} \otimes\left[\left(\overrightarrow{\boldsymbol{I}}_{p n_{f}}^{T} \otimes I_{\ell}\right) \cdot\left(I_{p n_{f}} \otimes \boldsymbol{\Theta}_{f}\right) \cdot\left(P_{j} \otimes I_{n_{f}}\right)\right]\right\} \cdot\left\{I_{L} \otimes\left[\left(I_{p n_{f}} \otimes \boldsymbol{\Theta}_{f}^{T}\right) \cdot\left(\overrightarrow{\boldsymbol{I}}_{p n_{f}} \otimes I_{\ell}\right)\right]\right\}\right\} .
\end{aligned}
$$

Note that $P_{j} \otimes I_{n_{f}} \in \mathbb{R}^{p n_{f} \times p n_{f}}$, and equals to $\left(P_{j} \otimes I_{n_{f}}\right) \otimes 1$; and $\Theta_{f} \in \mathbb{R}^{\ell p n_{f} \times 1}$. Therefore,

$$
\left(I_{p n_{f}} \otimes \boldsymbol{\Theta}_{f}\right) \cdot\left(P_{j} \otimes I_{n_{f}}\right)=\left(I_{p n_{f}} \otimes \boldsymbol{\Theta}_{f}\right) \cdot\left[\left(P_{j} \otimes I_{n_{f}}\right) \otimes 1\right]=\left(P_{j} \otimes I_{n_{f}}\right) \otimes \boldsymbol{\Theta}_{f} .
$$

Now by [1, Property (45)], (25) further reduces to

$$
\begin{aligned}
& \sum_{j=1}^{L} \mathbb{E}\left\{\left\{W_{j} \otimes\left[\left(\overrightarrow{\boldsymbol{I}}_{p n_{f}}^{T} \otimes I_{\ell}\right) \cdot\left(\left(P_{j} \otimes I_{n_{f}}\right) \otimes \boldsymbol{\Theta}_{f}\right)\right]\right\} \cdot\left\{I_{L} \otimes\left[\left(I_{p n_{f}} \otimes \boldsymbol{\Theta}_{f}^{T}\right) \cdot\left(\overrightarrow{\boldsymbol{I}}_{p n_{f}} \otimes I_{\ell}\right)\right]\right\}\right\} \\
= & \sum_{j=1}^{L} \mathbb{E}\left\{\left(W_{j} \cdot I_{L}\right) \otimes\left[\left(\overrightarrow{\boldsymbol{I}}_{p n_{f}}^{T} \otimes I_{\ell}\right) \cdot\left(\left(P_{j} \otimes I_{n_{f}}\right) \otimes \boldsymbol{\Theta}_{f}\right) \cdot\left(I_{p n_{f}} \otimes \boldsymbol{\Theta}_{f}^{T}\right) \cdot\left(\overrightarrow{\boldsymbol{I}}_{p n_{f}} \otimes I_{\ell}\right)\right]\right\} \\
= & \sum_{j=1}^{L} W_{j} \otimes\left\{\left(\overrightarrow{\boldsymbol{I}}_{p n_{f}}^{T} \otimes I_{\ell}\right) \cdot\left[\left(P_{j} \otimes I_{n_{f}}\right) \otimes \mathbb{E}\left(\boldsymbol{\Theta}_{f} \cdot \boldsymbol{\Theta}_{f}^{T}\right)\right] \cdot\left(\overrightarrow{\boldsymbol{I}}_{p n_{f}} \otimes I_{\ell}\right)\right\} \\
= & \sum_{j=1}^{L} W_{j} \otimes\left\{\left(\overrightarrow{\boldsymbol{I}}_{p n_{f}}^{T} \otimes I_{\ell}\right) \cdot\left[\left(P_{j} \otimes I_{n_{f}}\right) \otimes\left(\Omega \otimes \Sigma_{e}\right)\right] \cdot\left(\overrightarrow{\boldsymbol{I}}_{p n_{f}} \otimes I_{\ell}\right)\right\} \\
= & \left.\sum_{j=1}^{L} W_{j} \otimes\left\{\left(\overrightarrow{\boldsymbol{I}}_{p n_{f}}^{T} \otimes I_{\ell}\right) \cdot\left[\left(P_{j} \otimes I_{n_{f}} \otimes \Omega\right) \otimes \Sigma_{e}\right)\right] \cdot\left(\overrightarrow{\boldsymbol{I}}_{p n_{f}} \otimes I_{\ell}\right)\right\} \\
= & \sum_{j=1}^{L} W_{j} \otimes\left\{\left[\overrightarrow{\boldsymbol{I}}_{p n_{f}}^{T} \cdot\left(P_{j} \otimes I_{n_{f}} \otimes \Omega\right) \cdot \overrightarrow{\boldsymbol{I}}_{p n_{f}}\right] \otimes\left(I_{\ell} \cdot \Sigma_{e} \cdot I_{\ell}\right)\right\} \\
= & \sum_{j=1}^{L} W_{j} \otimes\left[\overrightarrow{\boldsymbol{I}}_{p n_{f}}^{T} \cdot\left(P_{j} \otimes I_{n_{f}} \otimes \Omega\right) \cdot \overrightarrow{\boldsymbol{I}}_{p n_{f}}\right] \otimes \Sigma_{e} .
\end{aligned}
$$

Here, the first and the second equality are due to [1, Property (45)]; the fourth one due to [1, Property (44)]; the fifth one again due to [1, Property (45)].

Now, by Lemma $4, \overrightarrow{\boldsymbol{I}}_{p n_{f}}^{T} \cdot\left(P_{j} \otimes I_{n_{f}} \otimes \Omega\right) \cdot \overrightarrow{\boldsymbol{I}}_{p n_{f}}=\operatorname{tr}\left(\Omega \cdot\left(P_{j}^{T} \otimes I_{n_{f}}\right)\right)=\operatorname{tr}\left(\left(P_{j} \otimes I_{n_{f}}\right) \cdot \Omega\right)$. The second equality is due to $\operatorname{tr}\left(M^{T}\right)=\operatorname{tr}(M)$. Thus, (25) finally boils down to

$$
\sum_{j=1}^{L} \operatorname{tr}\left(\left(P_{j} \otimes I_{n_{f}}\right) \cdot \Omega\right) \cdot\left(W_{j} \otimes \Sigma_{e}\right)
$$

On the other hand,

$$
\mathbb{E}\left\{\left\{I_{L} \otimes\left[\left(\overrightarrow{\boldsymbol{I}}_{p n_{f}}^{T} \otimes I_{\ell}\right) \cdot\left(I_{p n_{f}} \otimes \boldsymbol{\Theta}_{f}\right)\right]\right\} \cdot\left[\left(\sum_{j=1}^{L} W_{j}^{T} \otimes P_{j}^{T}\right) \otimes I_{n_{f}}\right] \cdot\left\{I_{L} \otimes\left[\left(I_{p n_{f}} \otimes \boldsymbol{\Theta}_{f}^{T}\right) \cdot\left(\overrightarrow{\boldsymbol{I}}_{p n_{f}} \otimes I_{\ell}\right)\right]\right\}\right\} .
$$

is the transpose of the matrix in (25), and hence equals to

$$
\sum_{j=1}^{L} \operatorname{tr}\left(\left(P_{j} \otimes I_{n_{f}}\right) \cdot \Omega\right) \cdot\left(W_{j}^{T} \otimes \Sigma_{e}\right) .
$$


Similarly,

$\mathbb{E}\left\{\left\{I_{L} \otimes\left[\left(\overrightarrow{\boldsymbol{I}}_{p n_{f}}^{T} \otimes I_{\ell}\right) \cdot\left(I_{p n_{f}} \otimes \boldsymbol{\Theta}_{f}\right)\right]\right\} \cdot\left(-I_{p n_{f} L}\right) \cdot\left\{I_{L} \otimes\left[\left(I_{p n_{f}} \otimes \boldsymbol{\Theta}_{f}^{T}\right) \cdot\left(\overrightarrow{\boldsymbol{I}}_{p n_{f}} \otimes I_{\ell}\right)\right]\right\}\right\}=-\operatorname{tr}(\Omega) \cdot\left(I_{L} \otimes \Sigma_{e}\right)$.

Now, substituting $(26,27,28)$ into (24), Eq. (20) follows. 